\title{
Low-threshold BBO OPO with cylindrical focusing
}

Sheng Wu, Geoffrey A. Blake, Sunny Sun, Jiwu Ling

Sheng Wu, Geoffrey A. Blake, Sunny Sun, Jiwu Ling, "Low-threshold BBO OPO with cylindrical focusing," Proc. SPIE 3263, Nonlinear Optical

Engineering, (19 May 1998); doi: 10.1117/12.308346

SPIE Event: Optoelectronics and High-Power Lasers and Applications, 1998, San Jose, CA, United States 


\title{
Low-threshold BBO OPO with cylindrical focusing
}

\author{
Sheng $\mathrm{Wu}^{\mathrm{a}}$, Geoffrey A. Blake ${ }^{\mathrm{b}}$ \\ Sunny Sun ${ }^{\mathrm{c}}$, Jiwu Ling ${ }^{\mathrm{c}}$ \\ Department of Chemsitry, California Institute of Technology, CA 91125 \\ Division of Geological and Planetary Sciences, California Institute of Technology, CA \\ 91125 \\ CASIX, Inc., CA 91016
}

\begin{abstract}
The design of a BBO OPO cavity based on cylindrical focusing of the pump beam in the insensitive plane of the non-linear crystal is presented and characterized. A variety of beam sizes in the sensitive plane of the BBO crystal are investigated, and in all cases this cavity design is found to significantly lower the operational threshold pulse energy of $355 \mathrm{~nm}$ pumped type I BBO OPOs. With optimal beam focussing parameters, the measured threshold intensity of $45 \mathrm{MW} / \mathrm{cm}^{2}$ is similar to that of conventional OPOs, but the threshold pulse energy of $0.4 \mathrm{~mJ}$ is some twenty times lower than that found in circularly symmetric pump beams. Pump pulse energies in this range can now be routinely achieved with diode-pumped Q-switched lasers, and the combination of these sources with cylindrically-focussed OPO cavities (COPOs) should result in a new class of all-solid-state high repetition rate and high average power non-linear light sources that can be tuned over the entire visible wavelength region.
\end{abstract}

Key words: BBO OPO, cylindrical focusing

Optical Parametric Oscillators (OPOs) based on BBO crystals have been demonstrated to be powerful, widely tunable solid state light sources in the visible and near-IR regions of the electromagnetic spectrum 1. However, because of its inherently large walk-off angle and small acceptance angle, BBO crystals are limited at present to studies that require high pulse energies and/or low repetition rates. For many scientific and industrial applications, average power and repetition rate are as or more important, and for such applications materials such as periodically-poled $\mathrm{LiNbO}_{3}$ have become increasingly important ${ }^{2}$. It is difficult, however, to pump periodically poled materials in the ultraviolet, and so to date there exist no robust high repetition rate counterparts to BBO-based optical OPOs that can be tuned over the transparency range of the nonlinear crystal (temperature tuned LBO OPOs do provide excellent low-threshold performance over the blue end of their $355 \mathrm{~nm}$-pumped tuning range, however ${ }^{3}$ ).

Numerically, previous experiments have shown that the lowest threshold pulse energy for BBO OPO is $1.7 \mathrm{~mJ} /$ pulse at $355 \mathrm{~nm}^{1}$. A number of all solid-state systems can produce pulses of this intensity, but such 
thresholds do precludes many diode pumped high power Nd:YAG lasers and even many compact flash lamp pumped Nd:YAG lasers, which have both small beam size and small pulse energies, from being used as pump sources for widely tunable visible wavelength OPOs.

To investigate these issues, we have constructed a $355 \mathrm{~nm}$-pumped OPO cavity based on a single type I BBO crystal $15 \mathrm{~mm}$ in length, with a broadband AR coating. The cavity is abbreviated COPO (for Cylindrical OPO) after the fact that we focus the pump beam with a cylindrical lens in the insensitive plane of the BBO crystal (see Figure 1). This cavity is based on the simple type II BBO standing wave design described by Wu et al. (1997) ${ }^{4}$. The pump beam delivery system has a cylindrical lens placed immediately before the OPO resonator whose focal point is inside the BBO crystal. As might be expected, precise positioning of the focal point of the cylindrical lens is required to optimize the OPO output power. The cylindrical lens is also mounted on a rotary mount in order to provide fine angular adjustment of the cylindrical lens after it was discovered that very small angular rotations of the cylindrical lens could extinguish the COPO output. This effect is a manifestation of and clearly demonstrates the large difference in acceptance angles of BBO crystals along their sensitive and insensitive directions.

A variety of tests were performed on the cavity presented in Figure 1 with both standard and cylindrical focusing optics, the results of which are summarized in Table 1. The cylindrical lens has a focal length of $200 \mathrm{~mm}$, and the pulse width of the injection seeded $355 \mathrm{~nm}$ Nd:YAG pump laser is about $10 \mathrm{~ns}$. Threshold energies of a conventional OPO are measured without the cylindrical lens, and for this arrangement a minimum threshold pulse energy of $8.5 \mathrm{~mJ}$ is found with a pump beam waist of $3 \mathrm{~mm}$. As shown in Table 1 , the optimum size of the pump beam before entering the cylindrical lens is also $3 \mathrm{~mm}$ in diameter. With cylindrical focussing, the pump beam remains about $3 \mathrm{~mm}$ in size in the sensitive direction inside the BBO crystal, which is large enough to overcome the walk-off effect through its $15 \mathrm{~mm}$ length. After being focused by the cylindrical lens, however, the size of the pump beam in the insensitive direction is only $\sim 30$ microns within the BBO crystal. This increases the intensity of the pump beam by a factor of $\sim 100$, and we have found that the threshold of the BBO COPO cavity is only $400 \mu \mathrm{J}$ when pumped at $355 \mathrm{~nm}-20$ times smaller than the conventional BBO OPO cavity. The threshold intensities presented in Table 1 were measured at a signal wavelength of $500 \mathrm{~nm}$, and lie within a factor of two of these values across the entire OPO tuning range of $430-2000 \mathrm{~nm}$.

Table 1 also presents the threshold values for a variety of pump beam waist sizes from 1.5 to $5.0 \mathrm{~mm}$. From this listing, it is apparent that walk-off effects begin to dominate the COPO's operational threshold when the beam size before passing through the cylindrical lens is smaller than $2.5 \mathrm{~mm}$ in diameter. Alternatively, an input beam size larger than $4 \mathrm{~mm}$ in diameter does not lower the operation threshold because the tighter focus also makes the depth of focus smaller, thereby reducing the effective interaction length of the parametric process. In between these extremes, the threshold pump pulse energy of the cavity varies by less 
than $10 \%$. Due to the cylindrically focused pump laser beam, the signal and idler output of the COPO expand very rapidly in the insensitive direction. An additional cylindrical lens is therefore placed outside the cavity to collimate the diverging output.

To summarize, an efficient, low-threshold $355 \mathrm{~nm}$-pumped bulk BBO OPO design is presented and characterized. The OPO layout, termed COPO, overcomes the walk-off effect of BBO crystals by maintaining a large beam size in the sensitive direction of the BBO crystal. Cylindrical focusing in the insensitive direction increases the pump intensity while avoiding walk-off losses, thereby lowering the pulse energy requirement of the pump laser. This design will allow many compact pulsed Nd:YAG lasers, as well as some high pulse energy diode pumped solid state lasers, to efficiently pump widely tunable BBO OPOs in the visible to near-IR wavelength range. The COPO cavity is also consistent with a variety of bandwidthnarrowing schemes such as grating feedback and injection seeding, which should lead to the development of compact, all solid state, high resolution spectroscopic sources that would find a great many uses in biology, chemistry, and the atmospheric/materials sciences.

\section{REFERENCES}

1. W.R. Bosenberg, L.K. Cheng, and C.L. Tang, Appl. Phys. Lett. 54, 13 (1989)

2. L.E. Myers, R.C. Eckardt, M.M. Fejer, R.L. Byer, Bosenberg, W.R, J.W. Pierce, J. Opt. Soc. Am. B, 12, 2102-2116 (1995)

3. D. Wang, C. Grasser, R. Bergaury, R. Wallenstein, Opt. Comm., 138, 87-90 (1997)

4. S. Wu, G.A. Blake, Z.Y. Sun, J.W. Ling, Appl. Optics 36, 5898-5901, (1997) 
Table 1. Operation threshold of COPO versus beam size at $500 \mathrm{~nm}$

\begin{tabular}{|c|c|c|c|c|c|c|c|}
\hline $\begin{array}{c}\text { Beam Size } \\
(\mathrm{mm})\end{array}$ & 1.5 & 2.0 & 2.5 & 3.0 & 3.5 & 4.0 & 5.0 \\
\hline COPO Threshold $(\mu \mathrm{J})$ & 3500 & 900 & 500 & 400 & 450 & 500 & 1200 \\
\hline $\begin{array}{c}\text { Threshold Intensity } \\
\left(\mathrm{MW} / \mathrm{cm}^{2}\right)\end{array}$ & 393 & 101 & 56 & 45 & 51 & 56 & 135 \\
\hline $\begin{array}{c}\text { Threshold Energy of } \\
\text { Conventional OPO } \\
(\mu \mathrm{J})\end{array}$ & 15,000 & 10.000 & 8.000 & 8.500 & 12.500 & 16.000 & 26.000 \\
\hline
\end{tabular}

The cylindrical lens has a focal length of $200 \mathrm{~mm}$, and the pulse width is about $10 \mathrm{~ns}$.

The threshold energy of a conventional OPO is measured without the cylindrical lens in Figure 1

Cylindrical lens

Mi

Mo, ouput

coupler

Cylindrical lens to collimate the signal/idler output<smiles>C1=CC=C1</smiles>

(1)

(1)
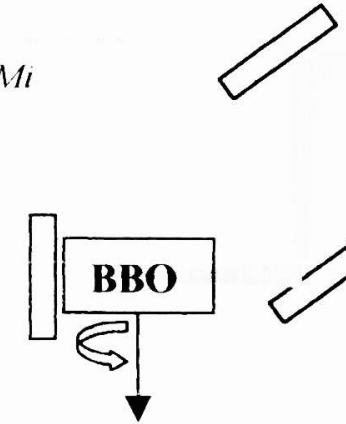

$\square$

Axis of rotation
U

\section{Pump beam}

Vertical polarization

$M r$, rear mirror

Figure 1. Mo is also a high reflector at pump wavelength, so we have double passing inside the cavity. Mr is a high reflector at either signal or idler wavelength. $M i$ is the pump reflector at 45 degrees. 